\title{
Didactics of Natural Sciences in Higher Secondary Education
}

\author{
Ana Cano Ortiz ${ }^{1}$, José Carlos Piñar Fuentes ${ }^{2}$, Eusebio Cano ${ }^{2 *}$ \\ ${ }^{1}$ Institute of Higher Secondary Education. Ministry of Education Madrid, Spain \\ ${ }^{2}$ Department. of Animal, Plant Biology and Ecology, Section of Botany, University of Jaen, Campus Universitario Las \\ Lagunillas s/n. 23071, Jaen, Spain
}

*Corresponding Author: Eusebio Cano, Department. of Animal, Plant Biology and Ecology, Section of Botany,
University of Jaen, Campus Universitario Las Lagunillas s/n. 23071, Jaen, Spain

Abstract: Didactic aspects are studied in the teaching of botanical subjects in secondary and high school education. We apply inductive, deductive and mixed methods, demonstrating the importance of the latter to connect the abstract with natural reality. In the case of the inductive, a vegetation profile is obtained, in which the student understands the biodiversity when ascending in altitude, while with the deductive one can only make an approximation to the interpretation of the territory, however when we use the mixed deductiveinductive method information is obtained that leads us to a real interpretation of the studied territory, in this case the Quercus suber forests

Keywords: Botany, Education, Methods, Plant communities, vegetation profiles, Cartography

\section{INTRODUCTION}

Los contenidos que se imparten en Ciencias Naturales están orientados a que los alumnos y las alumnas adquieran las bases propias de la cultura científica, haciendo especial hincapié en la unidad de los fenómenos que estructuran el ámbito natural, en las leyes que los rigen y en la expresión matemática de esas leyes, obteniendo con ello una visión racional y global de nuestro entorno con la que puedan afrontar los problemas actuales relacionados con la vida, la salud, el medio y las aplicaciones tecnológicas.

En la etapa de Educación Secundaria y Superior (Bachiller) se abordan los aspectos físico-químicos, biológico-geológicos de la naturaleza y del ser humano.

En la sociedad actual la ciencia es un instrumento indispensable para comprender el mundo que nos rodea y sus transformaciones, así como para desarrollar actitudes responsables sobre aspectos ligados a la vida, la salud, recursos naturales y medio ambiente.

Los conocimientos sobre ciencias de la naturaleza deben ser afianzados y ampliados durante la etapa secundaria y el bachillerato, mediante la incorporación de actividades prácticas, y adaptando los contenidos a la realidad social y ambiental del momento, por lo que es imprescindible incluir en los contenidos diversos aspectos ambientales, como es el caso de la contaminación de tierras, aguas, incendios, cambio climático. Esto permitirá. adoptar actitudes críticas fundamentadas en el conocimiento, para analizar individualmente o en grupo, cuestiones científicas y tecnológicas; desarrollar actitudes y hábitos favorables a la promoción de la salud personal y comunitaria, facilitando estrategias que permitan hacer frente a los riesgos de la sociedad actual, en aspectos relacionados con la alimentación, el consumo, las drogodependencias, la sexualidad y el medio ambiente. Comprender la importancia de utilizar los conocimientos de las ciencias de la naturaleza para satisfacer las necesidades humanas, y participar en la necesaria toma de decisiones en torno a problemas locales y globales a los que nos enfrentamos. Conocer y valorar las interacciones de la ciencia y la tecnología con la sociedad y el medio ambiente [1], con atención particular a los problemas a los que se enfrenta hoy la humanidad, y la necesidad de búsqueda y aplicación de soluciones, sujetas al principio de precaución, para avanzar hacia un futuro sostenible. Reconocer el carácter tentativo y creativo de las ciencias de la naturaleza, así como sus aportaciones al pensamiento 
humano a lo largo de la historia, apreciando los grandes debates superadores de dogmatismos y las revoluciones científicas que han marcado la evolución cultural de la humanidad y sus condiciones de vida.

Para ello se deben trabajar los contenidos sobre Biología y Geología, que deben ser actualizados debido al gran avance en investigación que ha sufrido la Biología y Geología en los últimos tiempos, respecto a los temas de salud (medicina) con el descubrimiento del mapa genético y células madre; así como el avance en el conocimiento de los recursos naturales y problemas medioambientales, catástrofes naturales y artificiales, cambio climático, justifica estas enseñanzas, que deben ser reforzadas en el curriculum del alumno [2].

Los objetivos generales y específicos para el aprendizaje que debemos poner de manifiesto para el desarrollo de capacidades del alumnado en el ámbito ambiental son:

1. Comprender y utilizar las estrategias y los conceptos básicos de las ciencias de la naturaleza para interpretar los fenómenos naturales, que permita adquirir el conocimiento y la interacción con el mundo físico.

2. Aplicar, en la resolución de problemas, estrategias coherentes con los procedimientos de las ciencias, tales como la discusión del interés de los problemas planteados, la formulación de hipótesis, la elaboración de estrategias de resolución y de diseños experimentales, el análisis de resultados, la consideración de aplicaciones y repercusiones del estudio realizado y la búsqueda de coherencia global.

3. Comprender y expresar mensajes con contenido científico utilizando el lenguaje oral y escrito con propiedad, interpretar diagramas, gráficas, tablas y expresiones matemáticas elementales, así como capacidad de comunicación, argumentaciones y explicaciones en el ámbito de la ciencia, lo que permitirá la adquisición de competencia lingüística, digital, matemática y ambiental.

\section{Metodología}

$\mathrm{El}$ aprendizaje se concibe como un cambio de esquemas conceptuales por parte de quien aprende. Se parte, pues, de la aceptación de que los alumnos poseen esquemas previos de interpretación de la realidad. Para ello es preciso una organización de los contenidos, que tenga presente la propia naturaleza de la ciencia como actividad constructiva y en permanente revisión. Construcción del conocimiento que se favorece mediante un aprendizaje activo, por lo que el alumno no puede ser un mero receptor pasivo.

De este modo, lo que se aprende depende fundamentalmente de lo ya aprendido (conocimientos previos), y por otro lado, quien aprende construye el significado de lo aprendido a partir de la propia experiencia; es decir, a partir de su actividad con los contenidos de aprendizaje y con su aplicación a situaciones similares.

El proceso de enseñanza-aprendizaje para las ciencias está formado por un conjunto de actividades incluidas en el ámbito del contenido, por ello la importancia de actualizar y adaptar el contenido del aprendizaje a la realidad social [3].

Desde el punto de vista didáctico es preciso utilizar para el aprendizaje diferentes métodos. a) Inductivo, en el que partiendo de lo cercano al alumno se pasa al conocimiento de lo global, es el caso de las experiencias que adquiere el alumno sobre la Biología y la Geología de campo, pudiendo ser este método utilizado en alumnado de la educación secundaria, el cual puede ser completado con la actitud indagatoria, activa, explicativa y participativa del alumnado, método más acorde con el carácter cognitivo de alumnos de secundaria. b) Deductivo, en este caso se parte de lo global para concluir en lo particular, método que permite interpretar el medio natural y que por su carácter abstracto, puede ser útil para el alumnado de educación superior (bachillerado y universidad), si bien como en el método inductivo es necesario completarlo con la actitud indagatoria, activa, explicativa y participativa. Para la aplicación del método inductivo hemos seleccionado un territorio concreto para su estudio; sin embargo para la aplicación del método deductivo se utilizan fotografías aéreas con delimitación de unidades de vegetación; estas unidades son posteriormente comprobadas en campo, lo que entronca ambos métodos, creando el método c) mixto. 


\section{RESUlTAdOS Y Discusión}

Como resultado de la aplicación de los diversos métodos de aprendizaje, el alumno adquiere diversas competencias. Relacionar la evolución de la ciencia con los avances en la lucha contra la explotación excesiva de los recursos naturales. Justificar razonadamente algunos fenómenos naturales, como la formación del suelo, influencia del paisaje vegetal en el modelado del relieve. Usar el lenguaje matemático para cuantificar el consumo de distintos recursos naturales, a fin de resolver problemas y realizar gráficos y tablas que permitan ordenar la información y extraer conclusiones. Uso de la tecnología de la comunicación y de la programación para la búsqueda de información digital, con aplicación de esta a diferentes campos del conocimiento [4], [5]. Adquisición de un lenguaje adecuado sobre la terminología científica en el campo de la Biología y de la Geología [6], [7]. La adquisición de estas competencias permiten al alumno adquirir una transversalidad [8], [9], al conectar los aspectos lingüísticos, matemáticos, digitales y tecnológicos con el aprendizaje de conceptos científicos [10]. Debido a las peculiaridades particulares de cada alumno, el profesor debe poner en práctica desde los niveles educativos inferiores hasta la universidad la acción tutorial, que debe ser personalizada, dicha acción permite un contacto directo con el alumno y la implicación de este en actividades de refuerzo y de ampliación del conocimiento, fundamentalmente mediante actividades prácticas de laboratorio, gabinete y campo, que motive al alumnado con proyección de imágenes y videos de distintos biomas de la Tierra: selvas, desiertos, praderas, taiga, tundra; o bien el estudio de comunidades de plantas, biodiversidad y bioclimatología [11], [12], [13] utilizando para ello el trabajo de campo, empleo de fotografías, videos y programas informáticos, como es [14] www.globalbioclimatic.org, [15], [16], [17], [18], [19], [20], [21].

El aprendizaje de los tipos de comunidades de plantas: bosques, matorrales, pastizales, son estudiadas por los alumnos de enseñanza secundaria y bachillerato siguiendo el método inductivo. Teniendo en consideración la composición florística de las comunidades vegetales con las especies dominantes, su diversidad, ecología, distribución biogeográfica y su dinámica catenal [22], para ello se realizan perfiles de campo y fotografías, que permitan posteriormente en el trabajo de gabinete la interpretación de la realidad, así el alumno ha sabido diferenciar los diferentes tipos de bosques, matorrales y pastizales [23], [24], [25].

Para el estudio de las grandes unidades de vegetación, como son los diversos biomas de la Tierra, o bien aquella vegetación de amplia distribución, se sigue el método deductivo, el uso de las fotografías aéreas con la delimitación de polígonos en el laboratorio, y su comprobación posterior de campo aúnan ambos métodos. En la figura 1 utilizando el método inductivo mediante el trabajo de campo, previa selección del territorio y estudio de las características geológicas, geomorfológicas, edáficas, climáticas, bioclimáticas y biogeográficas, se separan las comunidades de bosques climácicos de las comunidades edafoxéricas, que se localizan sobre roquedos [10], [26], [27].

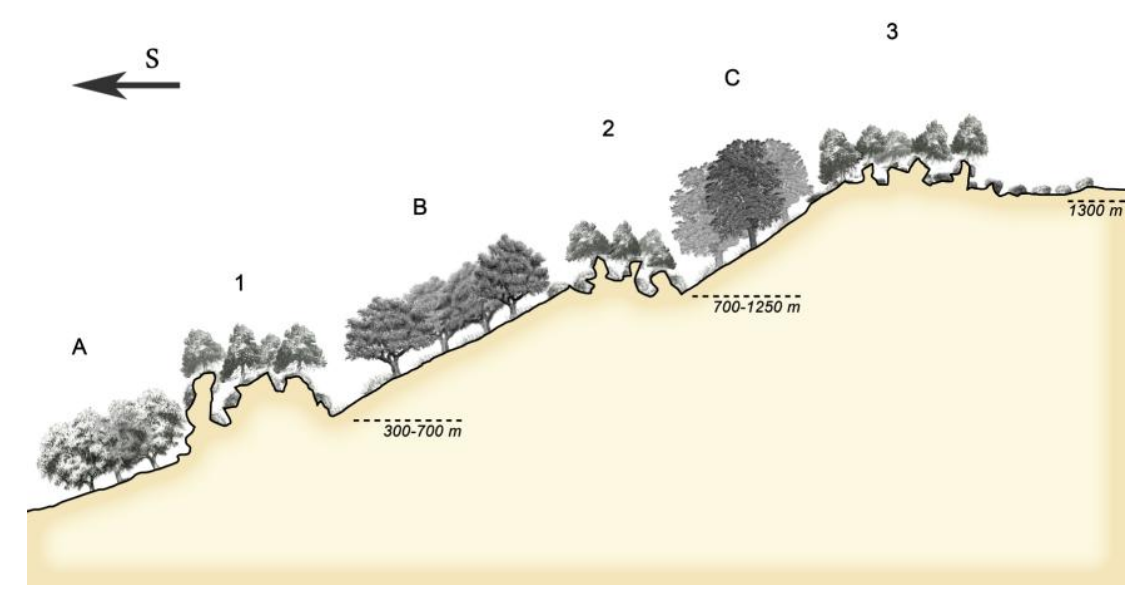

Figure1. A. Holm oaks. B. Cork oak forests. C. Robledales. 1. Thermophilic Juniperus. 2 and 3 Communities of Juniperus and Juniperus with Echinospartum in cold mountain environments.

La figura 2 ha sido obtenida a partir de fotografías aéreas y delimitación de polígonos de carácter uniforme en laboratorio, en este caso hemos seguido el método deductivo, estudiando de forma global el territorio, pero posteriormente se realizan las comprobaciones de campo para saber exactamente 
qué comunidad vegetal es, con esta actuación pasamos al método mixto (deductivo-inductivo). En este caso todos los polígonos corresponde a la misma comunidad vegetal, el alcornocal que se incluye en el hábitat de la UE 9330.

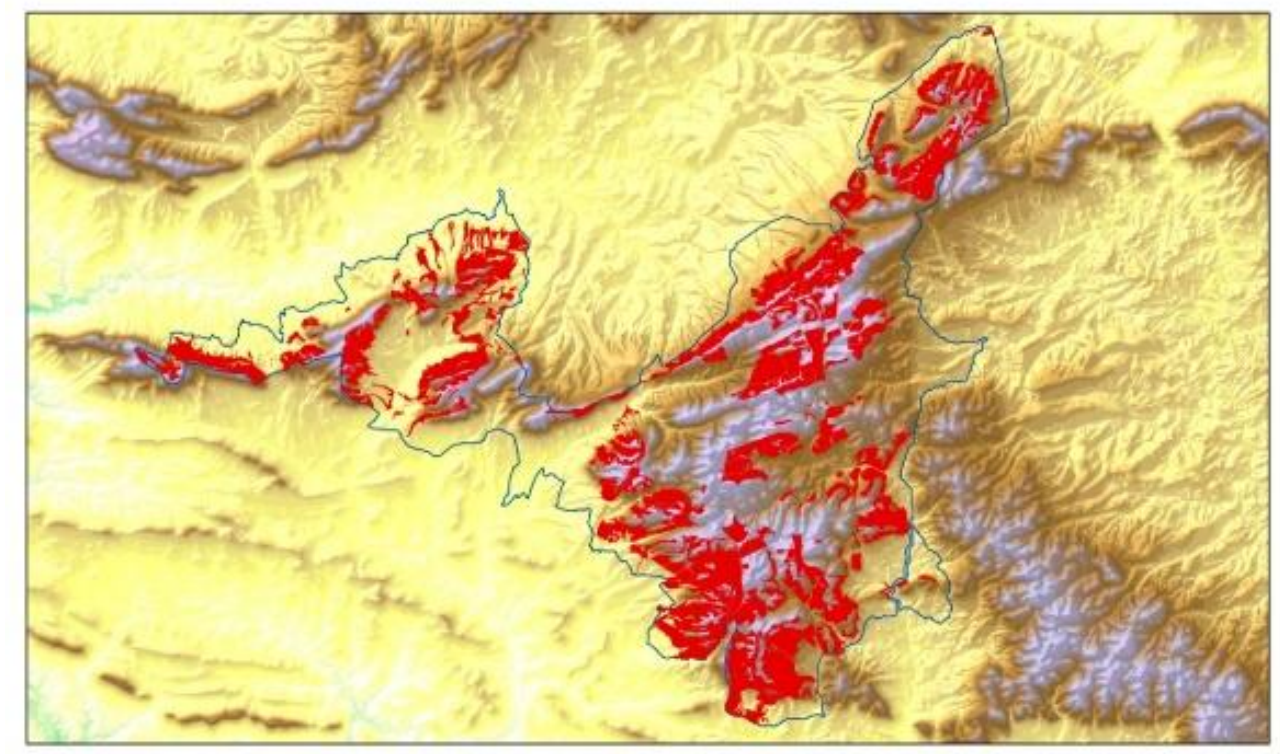

Figure2. Polygons in red. Cork oak forests, belonging to habitat 9330

\section{CONCLUSIONES}

La interpretación de la naturaleza puede hacerse desde varios métodos, inductivo, deductivo y mixto, siendo recomendable el método inductivo para alumnos de niveles inferiores (secundaria), y el deductivo y mixto para alumnos de bachiller y universidad. Se comprueba que las actividades prácticas de campo y laboratorio son fundamentales para la interpretación de la realidad natural. En el caso concreto del estudio botánico, el uso de perfiles o catenas de vegetación marcan la biodiversidad en cuanto al tipo de comunidades vegetales; en el caso del estudio global de la vegetación, se constata el valor de las fotografías aéreas y mapas a diferentes escalas.

\section{REFERENCIAS}

[1] Fernández, I. M, Pires, D. M. \& Villamañán, R. M. (2014). Educación científica con enfoque cienciatecnología-sociedad-ambiente: construcción de un instrumento de análisis de las directrices curriculares. Formación Universitaria, 7(5), 23-32. http://dx.doi.org/10.4067/S0718-50062014000500004.

[2] Bello Benavides, L.O., Cruz Sánchez, G.E., Meira Cartera, P.A. \& González Gaudiano, E. (2021). El cambio climático en el bachillerato. Aportes pedagógicos para su abordaje. Enseñanza de las Ciencias, 39-1, 137156. https//doi.org/10.5565/rev/ensciencias.3030.

[3] García Barros, S., Martínez Losada, C. \& Rivadulla López, J. (2021). Actividades de textos escolares. Su contribución al desarrollo de la competencia científica. Enseñanza de las Ciencias, 39-1, 219-238. https//doi.org/10.5565/rev/ensciencias.3099.

[4] Cano-Ortiz, A. Piñar Fuentes, J.C., Ighbareyeh, J.M.H., Quinto Canas, R. \& Cano, E. (2021). Aspectos Didácticos en la Enseñanza de Conceptos Geobotánicos. IJHSSE 8(4): 1-6. https://doi.org/10.20431/23490381.0804008

[5] Cano-Ortiz, A., Piñar Fuentes, J.C., Ighbareyeh, J.M.H., Quinto Canas, R. \& Cano, E. (2021). Didactic Aspects in the Teaching of Vegetation in Secondary and High School Education. IJHSSE 8(6): 1-7. https://doi.org/10.20431/2349-0381.0806002

[6] Miño, M. H., Toia, S.N., Pérez, G.M., Gutierrez, T.N., González Galli, L.M., Meinardi, E. M. (2021). Comparación del conocimiento metacognitivo sobre la lectura de textos de Biología entre estudiantes de la Ciudad de Buenos Aires. Revista Electrónica de Enseñanza de las Ciencias, vol. 20, № 1, 114-134.

[7] Tovar-Gálvez, J.C. (2921). Design of Intercultural Teaching Practices for Science Education based on evidence. Enseñanza de las Ciencias, 39-1, 99-115. https://doi.org/10.5565/rev/ensciencias.2891

[8] Cañal, P. (2012). ¿Cómo evaluar la competencia científica?. Investigación en la Escuela, 78, 5-17.

[9] Cañas, A., Martín-Díaz, M \& Nieda, J. (2007). Competencia en el conocimiento y la interacción en el mundo físico. La competencia científica. Madrid. Alianza Editorial. 
[10] Cano, E., Piñar Fuentes, J.C., Cano-Ortiz, A., Leiva Gea, F., Ighbareyeh, J.M.H., Quinto Canas, R.J., Rodríguez Meireles, C.I., Raposo, M., Pinto Gomes, C.J., Spampinato, G., del Río González, S. \& Musarella, C.M. (2021). Climatología, bioclimatología y cubiertas vegetales: Herramientas para mitigar el cambio climático in Symposium Internacionational ad Honorem Dei Prof. Salvador Rivas Martínez, León 1-3 septiembre. Pag. 44-45

[11] Cano-Ortiz, A. (2008). Bioclimatología y Cubiertas Vegetales en el olivar. Serv. Publ. Universidad de Jaén. 93 pág. en material didáctico para las asignaturas: "Diseño de Plantaciones y Técnicas de Cultivo" y “Bioindicadores y Cubiertas Vegetales en el Olivar". ISBN: 978-84-8439-398-6.

[12] Cano-Ortiz, A. (2016). Bioindicadores y cubiertas vegetales en el olivar in Nuevas Tendencias en Olivicultura. Serv. Publ. Univ. Jaén, 69-115. ISBN:978-84-8439-000-0

[13] Cano, E. \& Cano-Ortiz, A. (2016). Bioclimatología aplicada a la agronomía in Nuevas Tendencias en Olivicultura. Serv. Publ. Univ. Jaén, 7-64. ISBN:978-84-8439-000-0.

[14] Rivas-Martínez, S. \& Rivas-Sáenz, S. (2004). Worldwide Bioclimatic Classification System. Phytosociological Reserch Center, www.globalbiolimatics.org

[15] Cano, E. (2018a). Aspectos biogeográficos y bioclimáticos naturales. https://tv.ujaen.es/es/video/ 1779.html

[16] Cano, E. (2018b). Erosión como consecuencia de las técnicas de cultivo utilizadas. https://tv.ujaen.es/es/video/1783.html

[17] Cano, E. (2018c). Indicadores vegetales ombroclimáticos. https://tv.ujaen.es/es/video/1793.html

[18] Cano-Ortiz, A. (2018). Bioindicadores edáficos y metodología de toma de muestra. https://tv.ujaen.es/ es/video/1778.html.

[19] Cano-Ortiz, A., Piñar Fuentes, J.C. \& Cano, E. (2021). The use of technology for the teaching of foliar structures. V International virtual conference on educational research and innovation- CIVINEDU. September, 29-30 (in press.)

[20] Cano-Ortiz, A., Piñar Fuentes, J.C. \& Cano, E. (2021). Proposals for the learning of plant diversity. V International virtual conference on educational research and innovation - CIVINEDU. Septembaer, 29-30 (un press.),

[21] Beltrán Maza, G. (2018). Como plantar un olivo. https://tv.ujaen.es/es/video/1827.html.

[22] Lazare J.-J. (2009). Phytosociologíe dynamico-catenal et gestión de la biodiversité. Acta Bot. Gallica, 156(1), 49-61.

[23] Quinto Canas R., Mendes P., Cano Ortiz A., Musarella C.M. and Pinto Gomes C.J. (2018). Forest fringe communities of the southwestern Iberian Peninsula. Revista Chapingo Serie Ciencias Forestales y del Ambiente, 24(3), 415-434 (2018) doi: 10.5154/r.rchscfa.2017.12.072.

[24] Cano Ortiz, A., Musarella, C.M., Piñar, J.C., Veloz, A. \& Cano, E. (2015). The dry forest in the Dominican Republic. Plant Biosystems, 149(3), 451-472. http://dx.doi.org/10.1080/11263504. 2015.1040484

[25] Cano Ortiz, A., Musarella, C.M., Pinto Gomes, C.J., Piñar Fuentes, C. \& Cano, E. (2020). Phytosocioological Study, Diversity and Conservation Status of the Cloud Forest in the Dominican Republic. Plants, 9(6), 741; doi:10.3390/plants9060741. https://www.mdpi.com/2223-7747/9/6/741

[26] Cano, E., Musarella C.M., Cano Ortiz, A., Piñar Fuentes, J.C. \& Pinto Gomes C.J. (2016). Vegetation series as a basis for hábitats and species conservation: methodological aspect. Botanique, 1, 21-26.

[27] Meléndez, B y Fuster, J. Mª (1978). Geología. Ed. Paraninfo.

Citation: Ana Cano Ortizl et.al. " Didactics of Natural Sciences in Higher Secondary Education" International Journal of Humanities Social Sciences and Education (IJHSSE), vol 8, no.10, 2021, pp. 6-10. doi: https://doi.org/10.20431/2349-0381.0810002.

Copyright: (C) 2021 Authors. This is an open-access article distributed under the terms of the Creative Commons Attribution License, which permits unrestricted use, distribution, and reproduction in any medium, provided the original author and source are credited. 